\title{
GxE Effects on Tocopherol Composition of Oils from Very Old and Genetically Diverse Olive Trees
}

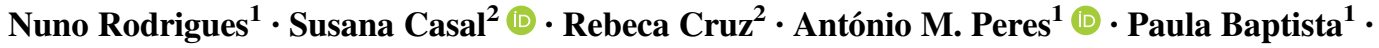 \\ José A. Pereira ${ }^{1}$ (i)
}

Received: 30 August 2019 / Revised: 15 December 2019 / Accepted: 30 January 2020

(C) 2020 AOCS

\begin{abstract}
Tocopherols are compounds with high biological activity, beneficial for human health that can be found in vegetable oils like olive oil, contributing for its resistance to oxidation. In this work, the tocopherol contents of olive oils extracted from centenarian olive trees of six cultivars (cvs. Lentisca, Madural, Rebolã, Redondal, Verdeal, and Verdeal Transmontana) were evaluated during five consecutive crop seasons (2013-2017). Three tocopherol isoforms $(\alpha-, \beta$ - and $\gamma$-tocopherols) were detected in all analyzed olive oils, and their content varied significantly with the cultivar and year of production. The highest amounts were found in $\mathrm{cv}$. Lentisca $(456 \pm 122 \mathrm{mg} / \mathrm{kg}$ olive oil), while the lowest were observed in cv. Verdeal $(179 \pm 45 \mathrm{mg} / \mathrm{kg}$ olive oil). Crop year was the most influential factor, with the highest contents observed in 2013 and lowest in 2014. Principal component analysis and hierarchical clustering analysis helped differentiate olive oils according to cultivar or production year. These data suggest that tocopherol composition may serve as a chemical marker to distinguish the subject cultivar olive oils from centenarian trees either by olive cultivar or by crop year, being some cultivars identified as potential candidates for guaranteeing the production of olive oils rich in these compounds.
\end{abstract}

Keywords Monovarietal olive oils $\cdot \alpha$-Tocopherol $\cdot$ Crop year · Vitamin E - Olive tree germplasm

José A. Pereira

jpereira@ipb.pt

1 Centro de Investigação de Montanha (CIMO), ESA, Instituto Politécnico de Bragança, Campus de Santa Apolónia, Bragança, 5300-253, Portugal

2 LAQV/REQUIMTE, Laboratory of Bromatology and Hydrology, Faculty of Pharmacy, University of Porto, Rua de Jorge Viterbo Ferreira, 228, Porto, 4050-313, Portugal
J Am Oil Chem Soc (2020).

\section{Introduction}

Virgin olive oil, obtained by mechanical and physical methods from fruits of the olive tree (Olea europaea L.), incorporates a huge chemical richness in terms of bioactive compounds (Boskou, 2015). Several studies showed that some of these microcomponents play important roles in human health (Huang and Sumpio, 2008; López-Miranda et al., 2010; Nocella et al., 2018; Visioli and Bernardini, 2011). Indeed, olive oil consumption has been associated with lower incidence rates of coronary disease and cancer, improved digestive functions, and brain aging delaying, among others (Abuznait et al., 2013; Huang and Sumpio, 2008; Nocella et al., 2018; Psaltopoulou et al., 2011; Visioli and Bernardini, 2011).

Among these bioactive molecules, vitamin E, the generic name used for a group of lipid compounds, including at least four different tocopherols and tocotrienols $(\alpha-, \beta-, \gamma-$ and $\delta$-) (Ahsan et al., 2015; Bramley et al., 2000) plays a key role in human health, acting as antioxidants in the lipid phase, capturing radicals in membranes, and lipoprotein particles (Esterbauer et al., 1991; Pryor, 2000). In particular, $\alpha$-tocopherol shows the highest biological activity, with numerous important clinical effects, regulating heme synthesis, inhibiting platelet aggregation, and participating in the prevention of degenerative neuropathies (Bramley et al., 2000; Pryor, 2000).

Vitamin $\mathrm{E}$ is typically found in vegetable sources with high lipid contents, as seeds and nuts, where they act primarily as antioxidants, protecting the integrity of the lipids and increasing their oxidative stability (Blekas et al., 1995; 
Bramley et al., 2000). In olive oil, $\alpha$-tocopherol is the predominant vitamin E compound, representing $90-95 \%$ in most cultivars (Kalogeropoulos and Tsimidou, 2017), whereas $\beta$ - and $\gamma$-tocopherols are only found in reduced amounts. However, the total amounts of vitamin $\mathrm{E}$ are variable, in a clear dependence of several factors, such as the cultivar, fruit ripening, environmental conditions, agronomic factors (e.g., irrigation, fertilization, pest, and diseases incidence), conditions used during oil extraction, storage, etc. (Aguilera et al., 2005; Beltrán et al., 2010; Cayuela and García, 2017; Kalogeropoulos and Tsimidou, 2017). Among all these factors, investigations using modern cultivars indicate that genotype has greatest influence on the contents and composition of tocopherols (GarcíaGonzález and Aparicio, 2010; Tura et al., 2007), but unfortunately this information remains unknown for most of the minor cultivars. Fruit ripening has some importance, with $\alpha$-tocopherol and total tocopherols decreasing during ripening (Beltrán et al., 2005). The edapho-climatic conditions are also recognized factors in olive oil compositional variability, in particular the year of production, which is known to significantly affect the content and composition of tocopherols due to stress conditions to which the plant is subjected throughout its reproductive cycle (Beltrán et al., 2010).

Recently, there has been an effort to find oils naturally rich in bioactive compounds. In this pursuit, some traditional olive cultivars may represent useful sources of bioactive compounds. Many genetically diverse centenarian olive trees can be found in the north of Portugal, especially in the region of Trás-os-Montes. Thus, this work aimed to characterize the tocopherol profile and contents in olive oils extracted from olives produced by centenarian trees belonging to six minor olive cultivars along five production years (2013-2017), with the purpose to explore their diversity and potential for future breeding, taking into account the olive oil with higher bioactive richness. To the Authors' best knowledge, information about the influence of genetic diversity on the tocopherol composition of olive oil is scarce in the literature, and has not been reported for the cultivars Lentisca, Rebolã, Redondal, and Verdeal.

\section{Materials and Methods}

\section{Sampling}

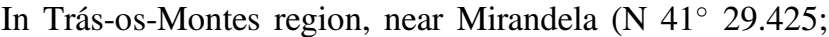
W $7^{\circ} 15.490$ ), Northeast of Portugal, one olive grove with centenarian trees was selected. According to the property records, the trees are more than 200 years old. Based on the tree appearance, structure, and trunk thickness, 20 trees of six minor cultivars were randomly selected, which included cvs. Lentisca (3 trees), Madural (3 trees), Rebolã (3 trees), Redondal ( 3 trees), Verdeal ( 2 trees), and Verdeal Transmontana (6 trees). During five consecutive crop seasons (from 2013 to 2017), approximately $3 \mathrm{~kg}$ of fruits were manually collected from each tree. To avoid the influence of the ripening stage on the olive oil composition, the harvest occurred when the fruits were between the ripening stage two and three that corresponds to the fruit epidermis with red spots in less than half the olive (MI 2) and the fruit epidermis red or purple in more than half the olive (MI 3). So, in every year the harvest occurred during the month of November, namely on the 25th and 26th days in 2013; on the 10th and 11th days in 2014; on the second and 3rd days in 2015; on the 7th and 8th days in 2016; and, on the 13th and 14th days in 2017. The fruits were extracted shortly after harvest, i.e., less than 24 hours after harvest, in a pilot extraction plant with an Abencor analyzer (Comercial Abengoa S.A., Seville, Spain), with three main units: a mill, a thermobeater where malaxation takes place at controlled temperature, and a centrifuge. Olives were milled, and the obtained paste was homogenized and about $700 \mathrm{~g}$ was transferred to the thermobeater unit $\left(25{ }^{\circ} \mathrm{C}, 20 \mathrm{~min}\right)$ for malaxation using a thermostatic water bath at $25{ }^{\circ} \mathrm{C}$. In the final $5 \mathrm{~min}$ of each malaxation, $100 \mathrm{~mL}$ of water at $27{ }^{\circ} \mathrm{C}$ was added to aid the olive oil separation. The mixture was centrifuged, decanted, and the olive oil collected. After that the oils were filtered (Whatman paper $\mathrm{n}^{\circ} 4$ ) using anhydrous sulfate in order to remove the solid particles and residual water. The olive oils were put in $125 \mathrm{~mL}$ dark bottles and stored in the dark at room temperature. All the assays were carried out between one and 2 months after extraction and were made in triplicate.

\section{Vitamin E Content and Profile}

Vitamin E contents were analyzed by high performance liquid chromatography (HPLC) with fluorescence detection, according to ISO 9936, 2006, with some modifications as detailed by Cruz and Casal (2013). Tocopherols standards ( $\alpha$, $\beta$, and $\gamma$ ) were purchased from Sigma (Spain), while the internal standard 2-methyl-2-(4,8,12-trimethyltridecyl)chroman6-ol (tocol) was from Matreya Inc. (Pleasant Gap, PA, USA). Individual standards purity was monitored by spectrophotometry (UV-1800, Shimadzu, Japan) based on their molar attenuation coefficients. N-hexane was HPLC grade from SigmaAldrich (Germany), 1,4-dioxane was from Sigma-Aldrich (PA, USA).

Filtered olive oil $(50 \mathrm{mg})$ was mixed with internal standard solution (tocol), diluted in n-hexane and homogenized. The mixture was centrifuged for $5 \mathrm{~min}$ at $13,000 \mathrm{rpm}$ at room temperature and the supernatant obtained analyzed by HPLC, using a normal phase silica column (Supelcosil ${ }^{\mathrm{TM}}$ LC-SI; 
$7.5 \mathrm{~cm} \times 3 \mathrm{~mm} ; 3 \mathrm{~mm}$ ) (Supelco, USA), conditioned at $25{ }^{\circ} \mathrm{C}$ and eluted with a mobile phase of 1, 4-dioxane in $\mathrm{n}$ hexane $(2.5 \%, \mathrm{v} / \mathrm{v})$, at a flow rate of $0.75 \mathrm{~mL} / \mathrm{min}$. Analyses were carried out using an integrated system with a data transmitter (Jasco LC - NetII/ADC, Japan), pumps (Jasco PU4180, Japan), an auto-sampler (Jasco AS - 4050, Japan), oven (ECOM Eco2000, Czech Republic), a DAD (Jasco MD - 4010, Japan), and fluorescence detector (FLD, Jasco FP-4025, Japan) programmed for excitation at $290 \mathrm{~nm}$ and emission at $330 \mathrm{~nm}$. Data were analyzed with the ChromNAV Control Center-JASCO Chromatography Data Station (Japan). The different compounds of vitamin $\mathrm{E}$ were identified by comparing the retention times with authentic standards, confirmed by their UV spectra and spectral purity and quantified by individual calibration curves, being expressed in $\mathrm{mg} / \mathrm{kg}$ of olive oil.

\section{Statistical Analysis}

One-way analysis of variance (one-way ANOVA) was applied to evaluate the existence of statistical significant effects of the tree cultivar or the crop year in the tocopherol contents of olive oils. Moreover, if a significant statistical effect was found $(P-$ value $<0.050$ ), the post-hoc multicomparison Tukey's test was also applied aiming to identify the levels (i.e., olive tree cultivar or crop year) of each effect that were responsible for the detected significant effect. Finally, boxplots were used to show the one-way ANOVA statistical results.

The possible influences of olive tree cultivar or crop year on the olive oil tocopherol profile was also evaluated using principal component analysis (PCA). For PCA, the tocopherols' contents were centered and scaled minimizing data variability.

Finally, hierarchical clustering heatmap was used to verify if the tocopherol profiles could be used as chemical markers to differentiate the olive oil obtained from olives picked from different cultivars. This false color image with dendograms is obtained by computing the distance (dissimilarity) between both rows (olive tree cultivar) and columns (tocopherol contents), being selected the Euclidean distance for matrix computation.

The statistical analysis was performed using the Subselect (Cadima et al., 2004; Cadima et al., 2012; Kuhn and Johnson, 2013) and MASS (Venables and Ripley, 2002) packages of the open source statistical program $\mathrm{R}$ (version 2.15.1), at a 5\% significance level.

\section{Results}

A total of 100 olive oils, from six cultivars, were extracted and analyzed during five consecutive crop years. In all the olive oil samples evaluated, three isoforms of tocopherols, namely $\alpha-, \beta$-, and $\gamma$-tocopherol, were identified and quantified. Their relative proportion was almost constant between years and cultivars, with $\alpha$-tocopherol ranging from 94.5 to $98.2 \%, \beta$-tocopherol from 0.4 to $1.9 \%$, and $\gamma$-tocopherol from 0.9 to $4.1 \%$, with average amounts of $96.8,1.1$, and $2.2 \%$, respectively. Fig. 1 shows a similar pattern of variation in absolute amounts ( $\mathrm{mg} / \mathrm{kg}$ of olive oil). For all olive cultivars evaluated, $\alpha$-tocopherol was the major isoform, varying between $118.4 \mathrm{mg} / \mathrm{kg}$ of olive oil (cv. Verdeal) and $607.1 \mathrm{mg} /$ $\mathrm{kg}$ of olive oil (cv. Lentisca). The ranking of $\alpha$-tocopherol contents by cultivar was: Lentisca $\approx$ Redondal $>$ Madural $\approx$ Rebolã $>$ Verdeal Transmontana $\approx$ Verdeal (Fig. 1). Indeed, the results pointed out that $\alpha$-tocopherol contents were significantly influenced by olive cultivar ( $P$-value $\leq 0.001$ for oneway ANOVA and/or Tukey's multicomparison test; Fig. 1).

The $\gamma$-tocopherol was the second isoform in terms of concentration, varying its contents between 1.4 (cv. Verdeal Transmontana) and $17.3 \mathrm{mg} / \mathrm{kg}$ of olive oil (cv. Lentisca). Similarly, the $\gamma$-tocopherol contents were significantly influenced by the olive cultivar ( $P$-value $\leq 0.001$ for oneway ANOVA and/or Tukey's multicomparison test; Fig. 1) in the following descending order: Lentisca $>$ Redondal $>$ Rebolã $\approx$ Madural $\approx$ Verdeal $>$ Verdeal Transmontana (Fig. 1). Finally, $\beta$-tocopherol was the isoform with the lowest amounts, varying from 1.0 (cv. Verdeal) to $7.5 \mathrm{mg} / \mathrm{kg}$ olive oil (cv. Lentisca) ( $P$-value $\leq 0.001$ for one-way ANOVA and/or Tukey's multicomparison test; Fig. 1). Considering the total content of vitamin $\mathrm{E}$ as the sum of the three abovementioned isoforms of tocopherols, the contents varied from 124.4 (cv. Verdeal) to $629.6 \mathrm{mg} / \mathrm{kg}$ of olive oil (cv. Lentisca), being these contents also significantly dependent of the olive cultivar ( $P$-value $\leq 0.001$ for one-way ANOVA and/or Tukey's multicomparison test; Fig. 1). The decreasing content order was the same as for $\alpha$-tocopherol.

From the overall tocopherol analysis in these six cultivars, it can be inferred that the centenarian cvs. Lentisca and Redondal are the most promising for obtaining olive oils rich in tocopherols.

These findings also helped to evaluate the possibility of using tocopherol composition to classify olive oils from centenarian trees. PCA suggested that the cultivars could be distinguished based on tocopherol composition using three principal components (PC), which explained $100 \%$ of the data variability $(82.3,11.7$ and $5.9 \%$ for $\mathrm{PC} 1, \mathrm{PC} 2$, and PC3, respectively) (Fig. 2).

For cvs. Lentisca and Redondal, $\beta$-tocopherol was the tocopherol that most contributed to the olive cultivars clustering. For cv. Madural, $\alpha$-tocopherol was the most influential isoform, whereas in cv. Rebolã, $\gamma$-tocopherol had the greatest influence. Overall, for the olive oils studied, the tocopherol content was highly dependent on the cultivar. The hierarchical clustering heatmap shown in Fig. 3 strengthen the previous 

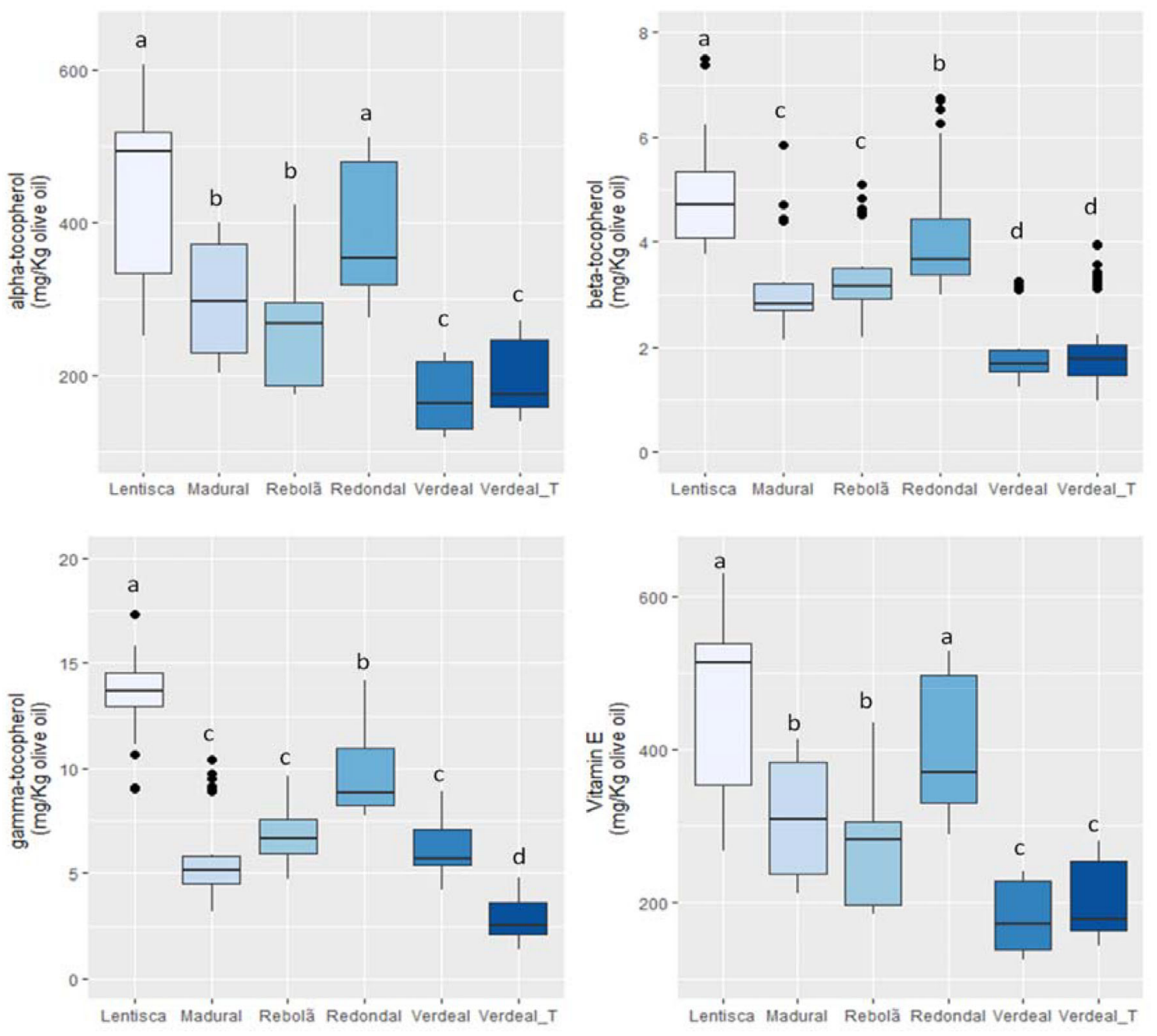

Fig. 1 Boxplots of the contents ( $\mathrm{mg} / \mathrm{kg}$ of olive oil) of $\alpha-, \beta$-, and $\gamma$-tocopherols as well as of vitamin E (total tocopherol content) found in olive oils extracted from olives collected from centenarian trees from different cultivars (cvs. Lentisca, Madural, Rebolã, Redondal, Verdeal, and Verdeal Transmontana) during five consecutive crop years (2013-2017). Different lowercase letters mean significant statistical differences at a 5\% significance level (one-way ANOVA followed by the Tukey's multicomparison test)

findings. Taking into account the dendograms information it is clear that the tocopherol contents may be split into two groups, one concerning the $\alpha$-tocopherol and the other comprising $\beta$ - and $\gamma$-tocopherols.

This information allowed grouping the olive oils into four main groups according to the olive cultivar. The first cluster mainly comprised olive oils from cvs. Lentisca, Madural, and Redondal (highest contents of $\alpha$-tocopherol and medium to high contents of the other two isoforms represented by yellow and light orange colors). The second cluster included cvs. Redondal, Madural, and Rebolã (medium to high contents of the three tocopherol isoforms, corresponding mainly to light orange and orange colors). The third and fourth clusters were mainly constituted by cvs. Verdeal and Verdeal Transmontana with some olive oils of cvs. Redondal and Madural (medium to low tocopherol contents, represented by orange and red colors). This analysis, although confirming that the olive cultivar greatly influenced the tocopherol contents of olive oils from centenarian trees, also pointed out that the crop year has also an important role, since the four identified clusters contained more than one cultivar.

Thus, considering that the climatic conditions vary from year to year, and that this work comprised five consecutive production campaigns, the effect of the crop year on the content of the different isoforms and the total of vitamin $\mathrm{E}$ was also evaluated, considering all the olive oils, regardless the olive cultivar. Fig. 4 shows the effect of the crop year on the content of the different tocopherol isoforms and of vitamin E. For $\alpha$-tocopherol, the highest levels were recorded in 2013 and 2015, being significantly higher $(P$ value $\leq 0.0056$, Tukey's multicomparison test) than those 


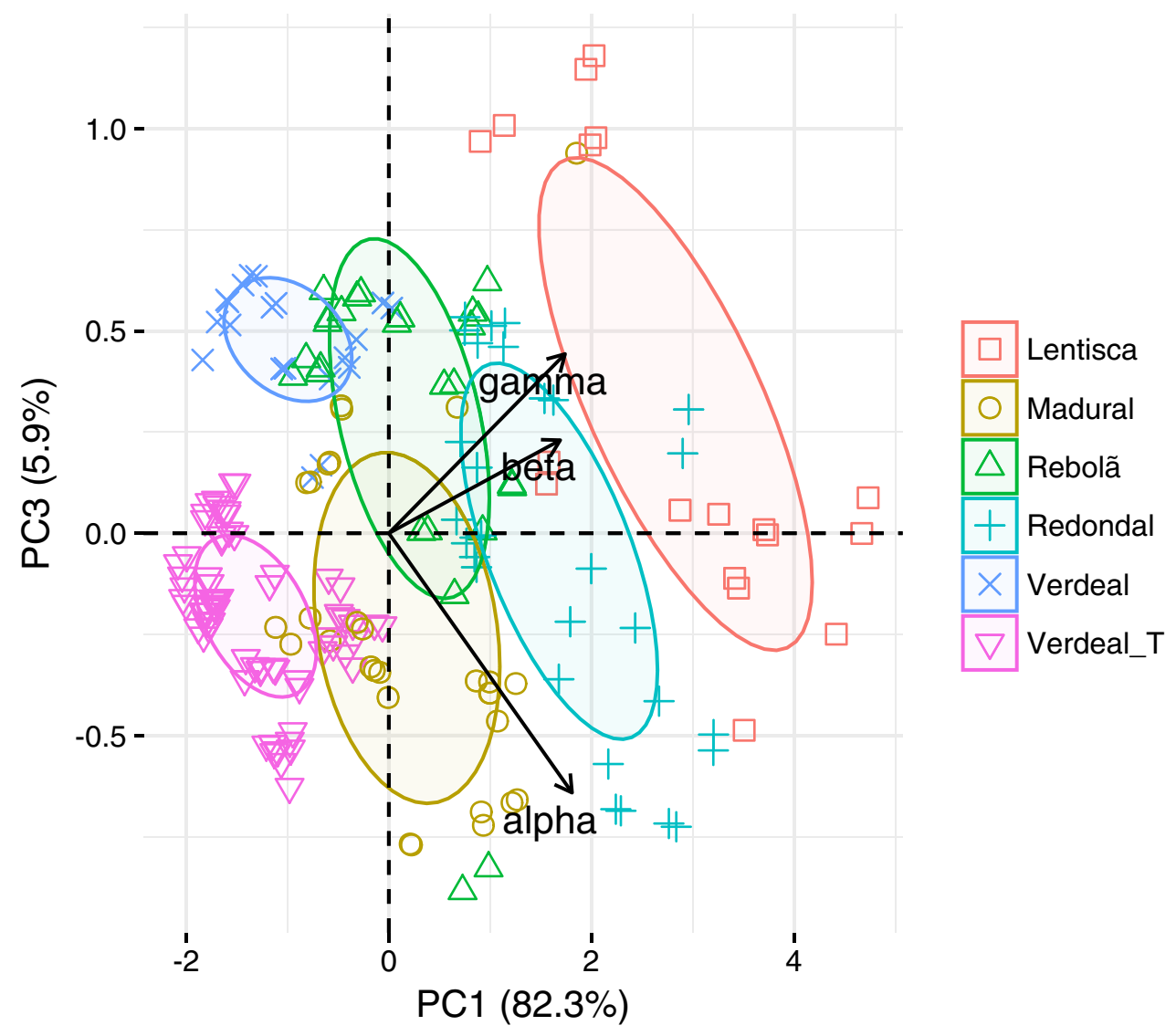

Fig. 2 Principal component analysis (PC1: 82.3\%, PC2: 11.7\%, and PC3: 5.9\%): 3D plot showing the unsupervised pattern recognition according to olive cultivar (Lentisca, Madural, Rebolã, Redondal, Verdeal, and Verdeal Transmontana) based on the $\alpha$-, $\beta$-, and $\gamma$-tocopherols contents found in olive oils obtained from olives collected from centenarian trees during five consecutive crop years (2013-2017)

determined for the other years. Thus, in decreasing order of abundance, $2013 \approx 2015>2017 \approx 2014 \geq 2016$ (Fig. 4). Similar to $\alpha$-tocopherol, the $\gamma$-tocopherol levels were significantly higher in $2013(8.7 \mathrm{mg} / \mathrm{kg}$ of olive oil) compared to the other crop years $(P$-value $\leq 0.0001$, Tukey's multicomparison test), while in 2014 it was observed the lowest average amounts $(4.1 \mathrm{mg} / \mathrm{kg}$ of olive oil). Also for $\beta$-tocopherol, statistically significant differences were observed between years ( $P$-value $\leq 0.0001$, Tukey's multicomparison test), with a higher significant content observed in 2015 ( $4.55 \mathrm{mg} / \mathrm{kg}$ of olive oil) (Fig. 4). For the total vitamin $\mathrm{E}$, in decreasing order of abundance, appeared $2013 \approx 2015>2017>2016 \approx 2014$ (Fig. 4). The analysis allowed verifying that the tocopherol profiles of olive oils from centenarian trees changed according to the crop year, independently of the olive cultivar. This conclusion was further confirmed by the PCA (Fig. 5). As can be observed, olive oils may be partially grouped according to the crop year (although some overlapping of samples from different years may be observed), being the year 2013, 2015, and 2016 the most differentiated.

\section{Discussion}

In general, the total contents of tocopherol found $(118.4-607.1 \mathrm{mg} / \mathrm{kg})$, as well as those of the different isoforms, are in agreement with the literature values although, in some cases, were higher (Beltrán et al., 2010; Borges et al., 2017; Noorali et al., 2017; Tura et al., 2007). In this work, $\alpha$-tocopherol represented more than $94 \%$ of the total tocopherol content in all the evaluated olive oils, which was also in accordance with the literature (Beltrán et al., 2010).

The olive oils studied were obtained from centenarian trees of six olive cultivars, namely cvs. Lentisca, Madural, Rebolã, Redondal, Verdeal, and Verdeal Transmontana, significantly differing the tocopherol amounts according to the olive cultivar (Fig. 1). The dissimilarity of the tocopherol amounts also allowed identifying patterns within the olives oils that enabled the oil differentiation and clustering by cultivar (Figs. 2 and 3).

These results further support the evidence that genetic factors (e.g., cultivar) have a great influence on the 

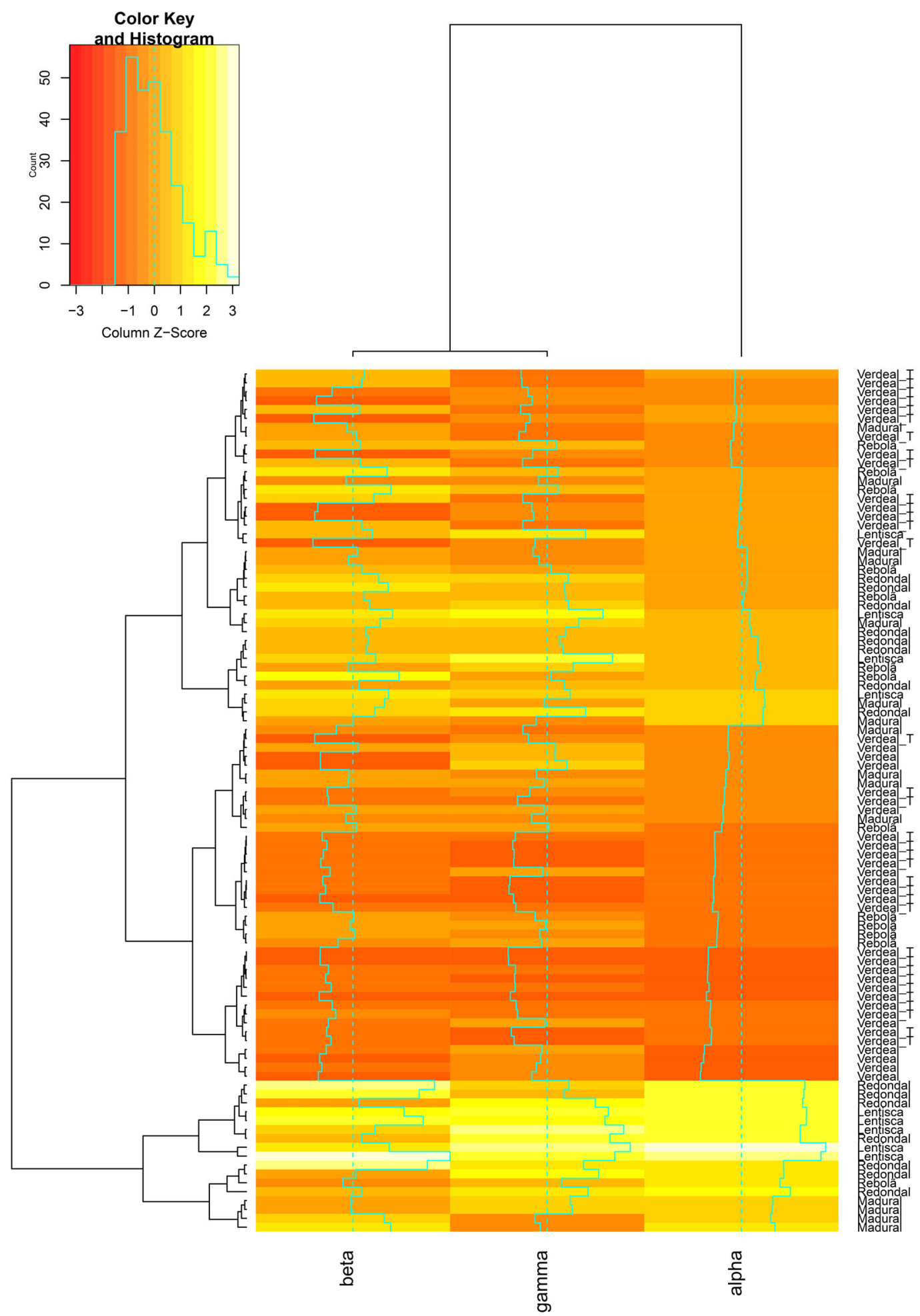

Fig. 3 Hierarchical clustering heatmap (using Euclidean distances and Ward method) and respective dendograms for variables ( $\alpha$-, $\beta$-, and $\gamma$-tocopherols contents) and olive oils obtained from centenarian olive trees of different cultivars (Lentisca, Madural, Rebolã, Redondal, Verdeal, and Verdeal Transmontana), during five consecutive crop seasons (2013-2017)

tocopherol concentrations found in olive oils. A study conducted with varietal olive oils from 18 Italian cultivars (Tura et al., 2007), showed that the total tocopherol contents, varying from 39.4 to $425.9 \mathrm{mg} / \mathrm{kg}$, were mainly dependent on the olive cultivar, having the environmental condition lower or no significance. In the same way, the 

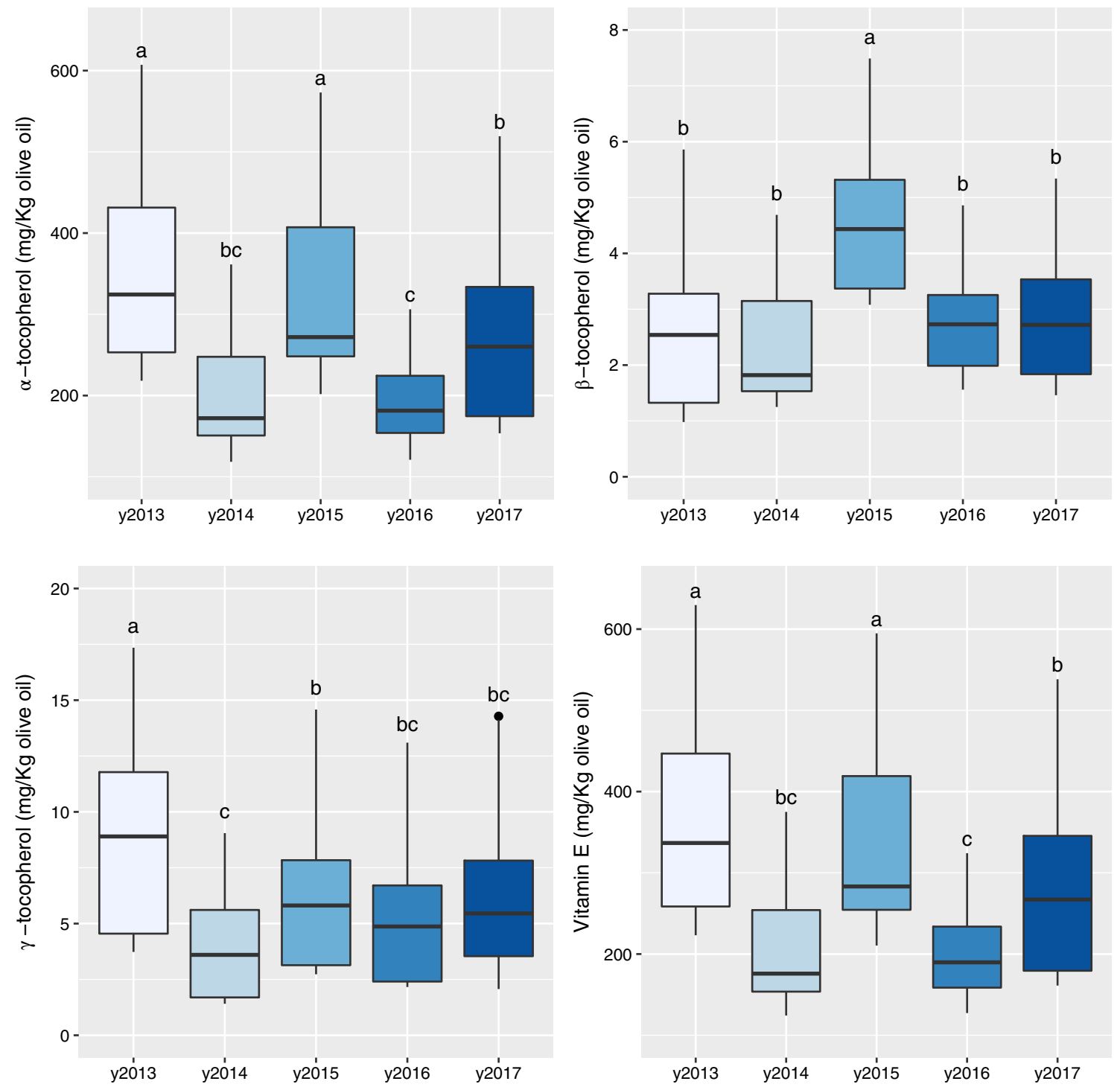

Fig. 4 Boxplots of the contents ( $\mathrm{mg} / \mathrm{kg}$ of olive oil) of $\alpha$-, $\beta$-, and $\gamma$-tocopherols as well as of vitamin $\mathrm{E}$ (total tocopherol content) found in olive oils extracted from olives collected from centenarian trees from different cultivars (cvs. Lentisca, Madural, Rebolã, Redondal, Verdeal, and Verdeal Transmontana) during five consecutive crop years (2013-2017). Different lowercase letters mean significant statistical differences at a 5\% significance level (one-way ANOVA followed by the Tukey's multicomparison test)

results from a study comprising 30 Spanish olive cultivars, with total tocopherol contents ranging from 84 to $463 \mathrm{mg} / \mathrm{kg}$ (Beltrán et al., 2010), also showed that the amounts of tocopherols in virgin olive oil were significantly affected by genetics or cultivar. Other works carried out in different countries such as Brazil (Borges et al., 2017), China (Xiang et al., 2017), Croatia (Špika et al., 2016), Greece (Boskou et al., 2006), Italy (Aguilera et al., 2005; Condelli et al., 2015), Spain (Beltrán et al., 2010; Franco et al., 2014), Tunisia (Laroussi-Mezghani et al., 2016), and Turkey (Arslan and Schreiner, 2012; Uluata et al., 2016), reported similar tocopherol ranges.
Concerning Portuguese olive oils, a huge amount of information is available for one of the most spread olive cultivar, cv. Cobrançosa, with reported amounts of 99-313 $\mathrm{mg} / \mathrm{kg}$ of olive oils, being these values dependent from the storage conditions of the olive fruits before extraction (Pereira et al., 2002), olive fly attack (Pereira et al., 2004), decreasing along the fruit ripening (Matos et al., 2007), and increasing with the addition of olive leaves during extraction (Malheiro et al., 2013). Also, Peres et al. (2016) reported that olive oils extracted from olives of cv. Cobrançosa and other typical cultivar (cv. Galega Vulgar), picked in early stages of ripening, had different tocopherol contents, but in lower amounts compared to 


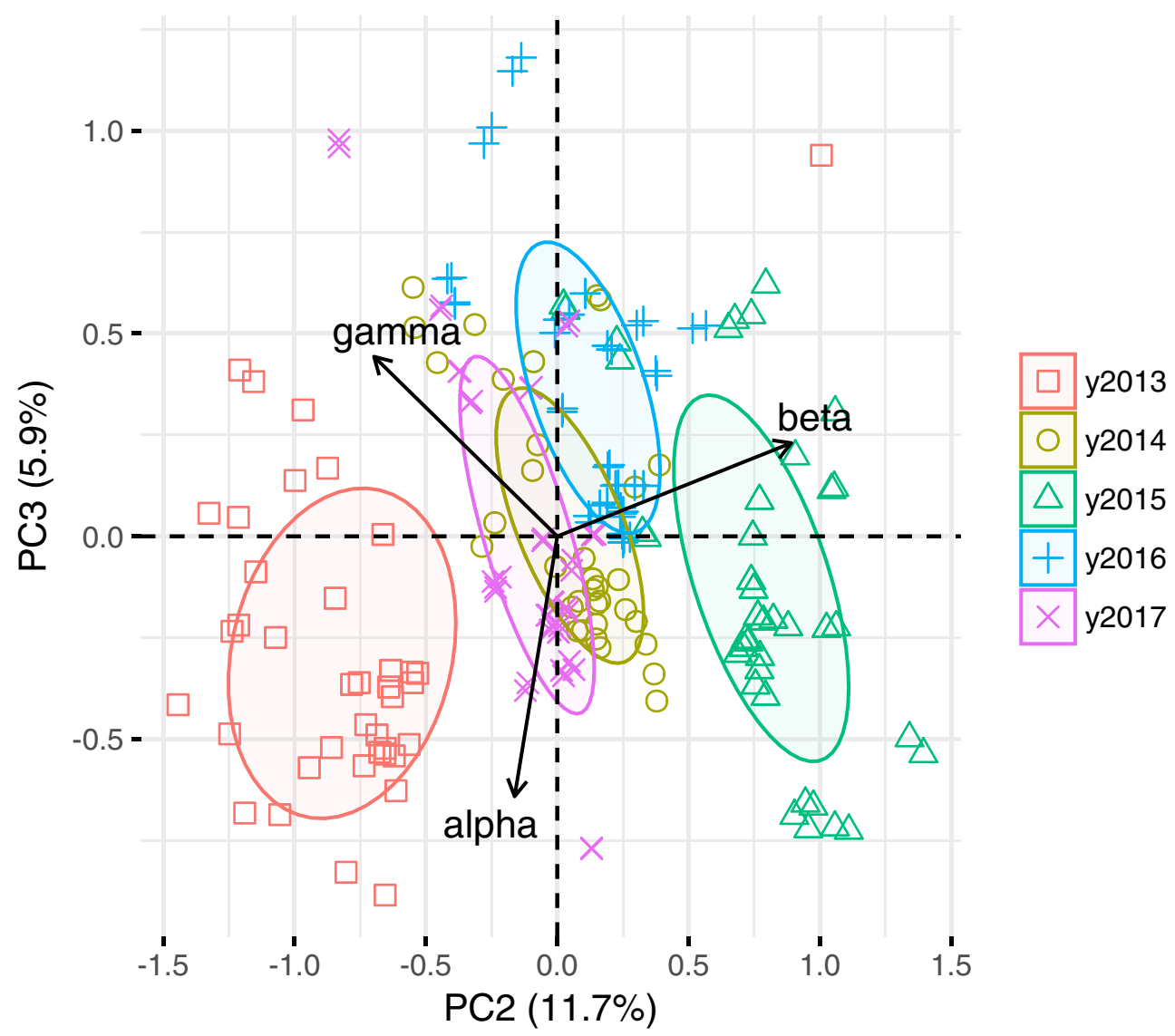

Fig. 5 Principal component analysis (PC1: 82.3\%, PC2: 11.7\%, and PC3: 5.9\%): 3D plot showing the unsupervised pattern recognition according to crop year (2013-2017) based on the $\alpha$-, $\beta$-, and $\gamma$-tocopherols contents found in olive oils obtained from olives collected from centenarian trees of different cultivars (Lentisca, Madural, Rebolã, Redondal, Verdeal, and Verdeal Transmontana)

those determined in this work for cvs. Lentisca, Madural, and Redondal. Garcia et al. (2012) studied four typical cultivars (cvs. Cordovil, Carrasquinha, Verdeal, and Negrinha de Freixo), with amounts within the expected range, from $201 \mathrm{mg} / \mathrm{kg}$ (cv. Negrinha do Freixo) to $391 \mathrm{mg} / \mathrm{kg}$ (cv. Cordovil). Additionally, these authors showed that there was a reduction trend with fruit ripening. Aware of these variations, we have eliminated this variability factor by using olives under the same ripening stage, in order to guarantee that the variations observed were strictly due to cultivar differences.

Although the effect of tree age on the oils composition was not an objective on this study, information available on this subject is scarce. There are many difficulties to develop and design a rigorous detailed study for this purpose, once it is not easy to assure the same genetic source of the plant material between different geographical origins. For a correct comparison, all trees should grow under the same edapho-climatic conditions and subjected to the same agronomic practices, being difficult to find groves under these conditions. Still, Chtourou et al. (2017) attempted to study the effect of tree age on the chemical composition of olive oils produced from a minor Tunisian cultivar, cv. Oueslati, under different ripening stages. The authors denoted some differences between adult and young trees, with a slight increase in total tocopherols in the older ones, but the effect of fruit ripening was more pronounced than tree age. To avoid this confounder, this study only used olive oils extracted from olives at the same ripening stage, being all trees fully adult ones.

There is a general consensus that the composition of olive oils varies from year to year, as a function of the climate conditions. However, most observations are only supported by studies conducted during 2 or 3 years. In the present study, with five consecutive crops evaluated, a clear interannual variation was observed. Beltrán et al. (2010) attributed these variations to rainfall levels, with higher tocopherol amounts in oils from drier crop years, although this trend was not observed in the present study (Table 1).

The content of vitamin $\mathrm{E}$ in olive oils may be included as part of the health claims allowing that olive oils to be labeled as "Source of Vitamin E," under certain conditions (levels established in the European Regulation EU 1169/2011, 2011). According to this regulation, and considering the 
Table 1 Minimum, mean, and maximum temperature $\left({ }^{\circ} \mathrm{C}\right)$ in the month of August, September, and October, year 2013-2017, Mirandela, Portugal

\begin{tabular}{|c|c|c|c|c|c|c|}
\hline & Month & 2013 & 2014 & 2015 & 2016 & 2017 \\
\hline \multirow[t]{3}{*}{ Minimum temperature $\left({ }^{\circ} \mathrm{C}\right)$} & August & $16.8 \pm 2.2$ & $14.3 \pm 2.6$ & $14.5 \pm 2.6$ & $15.8 \pm 2.4$ & $15.0 \pm 2.8$ \\
\hline & September & $14.2 \pm 2.2$ & $14.4 \pm 4.5$ & $11.9 \pm 3.0$ & $12.9 \pm 2.8$ & $10.8 \pm 3.1$ \\
\hline & October & $11.0 \pm 3.8$ & $10.9 \pm 3.0$ & $9.8 \pm 3.3$ & $9.9 \pm 2.7$ & $8.9 \pm 3.7$ \\
\hline \multirow[t]{3}{*}{ Mean temperature $\left({ }^{\circ} \mathrm{C}\right)$} & August & $25.6 \pm 9.3$ & $22.7 \pm 8.9$ & $23.2 \pm 9.4$ & $25.1 \pm 9.9$ & $24.4 \pm 10.0$ \\
\hline & September & $22.4 \pm 8.8$ & $21.0 \pm 7.6$ & $19.5 \pm 8.3$ & $21.4 \pm 9.6$ & $19.9 \pm 9.6$ \\
\hline & October & $16.4 \pm 6.4$ & $17.1 \pm 6.8$ & $15.2 \pm 6.2$ & $16.3 \pm 7.2$ & $17.7 \pm 10.0$ \\
\hline \multirow[t]{3}{*}{ Maximum temperature $\left({ }^{\circ} \mathrm{C}\right)$} & August & $34.4 \pm 3.4$ & $31.2 \pm 2.6$ & $31.9 \pm 4.4$ & $34.5 \pm 3.2$ & $33.8 \pm 3.4$ \\
\hline & September & $30.5 \pm 3.8$ & $27.6 \pm 4.5$ & $27.1 \pm 3.5$ & $30.0 \pm 5.5$ & $28.9 \pm 3.1$ \\
\hline & October & $21.9 \pm 2.9$ & $23.2 \pm 3.0$ & $20.6 \pm 2.7$ & $22.6 \pm 3.8$ & $26.5 \pm 5.4$ \\
\hline \multirow[t]{4}{*}{ Rainfall (mm) } & August & 0.0 & 0.4 & 20.2 & 32.2 & 1.2 \\
\hline & September & 30.0 & 84.8 & 50.4 & 24.2 & 0.2 \\
\hline & October & 111.4 & 77.2 & 109.4 & 41.6 & 28.6 \\
\hline & Sum & 141.4 & 162.4 & 180.0 & 98.0 & 30.0 \\
\hline
\end{tabular}

recommendation of a daily intake of $12 \mathrm{mg}$, the ingestion of $28.5 \mathrm{~mL}$ of olive oil from centenarian trees of cv. Lentisca (evaluated in the present work) would be sufficient to meet vitamin $\mathrm{E}$ daily intake requirements. For the other cultivars, higher amounts would be required, namely $32.3 \mathrm{~mL}$ for cv. Redondal, $41.9 \mathrm{~mL}$ for cv. Madural, $48.4 \mathrm{~mL}$ for cv. Rebolã, $63.4 \mathrm{~mL}$ for cv. Verdeal Transmontana and $72.8 \mathrm{~mL}$ for $\mathrm{cv}$. Verdeal. These values are of the same order of those reported in the literature, namely by Bayram et al. (2012), where a daily intake of $50 \mathrm{~mL}$ of olive oil was needed to meet the daily requirements of vitamin E. Although other vegetable oils can be regarded as richer sources of vitamin $\mathrm{E}$, particularly sunflower oil, it should be retained that the tocopherols are only a part of the rich antioxidant pool of virgin olive oils. The simultaneous ingestion of other olive oil hydrophilic phenolic compounds extracted from the olive fruit, such as the hydroxityrosol derivatives as well as monounsaturated fatty acids contribute also to the richness of olive oil. Still regarding tocopherols, it should also strengthen the olive oil richness in $\alpha$-tocopherol, the most bioactive vitamin $\mathrm{E}$ isoform. Other isoforms show less clear positive associations for some health effects, namely $\gamma$-tocopherol (CookMills and Mc Cary, 2010), the main isoform in several vegetables oils, as soybean oil. In this sense, the search for the highest contents in these bioactive compounds has shown that some cultivars are stronger candidates for obtaining olive oils with higher health promoting activities and higher oxidative stability.

\section{Conclusions}

The work carried out showed that olive oils obtained from olives collected from centenarian trees of different cultivars (cvs. Lentisca, Madural, Rebolã, Redondal, Verdeal and Verdeal Transmontana) may be a good source of tocopherols, and a daily intake of $28.5-72.5 \mathrm{~mL}$ (depending on the cultivar) would guarantee the daily needs for vitamin E. Indeed, from a health point of view, olive oils from cv. Lentisca were the most promising ones. Overall, three tocopherol isoforms ( $\alpha$-, $\beta$ - and $\gamma$-tocopherol) were found in all the olive oils, being $\alpha$-tocopherol the main isoform. Both olive cultivar and crop year had a significant influence on the tocopherol profiles. Hopefully, the results reported in this work will attract the attention and increase the interest of olive producers to these minor and almost forgotten cultivars that are still grown in the region of Trás-osMontes (Portugal). In fact, this study contributed to a better understanding of the genetic heritage that exists in this Portuguese region, showing the possibility of selecting and propagating some of these minor olive cultivars with the aim of obtaining differentiated olive oils rich in antioxidant compounds.

Acknowledgments This work was financially supported by Project UIDB/00690/2020 (CIMO) and Project UID/QUI/50006/2019 (REQUIMTE-LAQV); all funded by Fundo Europeu de Desenvolvimento Regional (FEDER) through COMPETE2020-Programa Operacional Competitividade e Internacionalização (POCI) and by national funds through Fundação para a Ciência e a Tecnologia (FCT), Portugal. This work was also supported by PRODER project OliveOld-Identificação e caraterização de oliveiras centenárias para obtenção de produtos diferenciados" $\mathrm{n}^{\circ}$ 53988. Nuno Rodrigues thanks FCT, POPH-QREN and FSE for the PhD Grant (SFRH/ $\mathrm{BD} / 104038 / 2014)$.

\section{References}

Abuznait, A. H., Qosa, H., Busnena, B. A., El Sayed, K. A., \& Kaddoumi, A. (2013) Olive-oil-derived Oleocanthal enhances 
$\beta$-amyloid clearance as a potential Neuroprotective mechanism against Alzheimer's disease: in vitro and in vivo studies. ACS Chemical Neuroscience, 4:973-982.

Aguilera, M. P., Beltrán, G., Ortega, D., Fernández, A., Jiménez, A., \& Uceda, M. (2005) Characterisation of virgin olive oil of Italian olive cultivars: "Frantoio" and "Leccino," grown in Andalusia. Food Chemistry, 89:387-391.

Ahsan, H., Ahad, A., \& Siddiqui, W. A. (2015) A review of characterization of tocotrienols from plants oils and foods. Journal of Chemical Biology, 8:45-59.

Arslan, D., \& Schreiner, M. (2012) Chemical characteristics and antioxidant activity of olive oils from Turkish varieties grown in Hatay province. Scientia Horticulturae, 144:141-152.

Bayram, B., Esatbeyoglu, T., Schulze, N., Ozcelik, B., Frank, J., \& Rimbach, G. (2012) Comprehensive analysis of polyphenols in 55 extra virgin olive oils by HPLC-ECD and their correlation with antioxidant. Plant Foods for Human Nutrition, 67:326-336.

Beltrán, G., Aguilera, M. P., Del Rio, C., Sanchez, S., \& Martinez, L. (2005) Influence of fruit ripening process on the natural antioxidant content of Hojiblanca virgin olive oils. Food Chemistry, 89: 207-215.

Beltrán, G., Jiménez, A., Del Rio, C., Sánchez, S., Martínez, L., Uceda, M., \& Aguilera, M. P. (2010) Variability of vitamin E in virgin olive oil by agronomical and genetic factors. Journal of Food Composition and Analysis, 23:633-639.

Blekas, G., Tsimidou, M., \& Boskou, D. (1995) Contribution of $\alpha$-tocopherol to olive oil stability. Food Chemistry, 52:289-294.

Borges, T. H., López, L. C., Pereira, J. A., Cabrera-Vique, C., \& Seiquer, I. (2017) Comparative analysis of minor bioactive constituents (CoQ10, tocopherols and phenolic compounds) in Arbequina extra virgin olive oils from Brazil and Spain. Journal of Food Composition and Analysis, 63:47-54.

Boskou, D. (2015) Olive and Olive Oil Bioactive Constituents (1st ed.). Champaign, IL: AOCS Press.

Boskou, D., Blekas, G., \& Tsimidou, M. (2006) Olive oil composition. In D. Boskou (Ed.), Olive oil, chemistry and technology (pp. 41-72). Champaign, IL: AOCS Press.

Bramley, P. M., Elmadf, I., Kafatos, A., Kelly, F. J., Manios, Y., Roxborough, H. E., ... Wagner, K. H. (2000) Vitamin E. Journal of the Science of Food and Agriculture, 80:913-938.

Cadima, J., Cerdeira, J. O., \& Minhoto, M. (2004) Computational aspects of algorithms for variable selection in the context of principal components. Computational Statistics \& Data Analysis, 47: 225-236.

Cadima, J., Cerdeira, J.O., Silva, P.D., \& Minhoto, M. (2012). The subselect $R$ package. http://cran.rproject.org/web/packages/ subselect/vignettes/subselect.pdf.

Cayuela, J. A., \& García, J. F. (2017) Sorting olive oil based on alpha-tocopherol and total tocopherol content using near-infra-red spectroscopy (NIRS) analysis. Journal of Food Engineering, 202: $79-88$.

Chtourou, F., Valli, E., Bendini, A., Aida, L., Toschi, T. G., \& Bouaziz, M. (2017) Effects of olive trees age on the minor components of Oueslati virgin olive oils produced from olives harvested at different ripening degrees. Journal of the American Oil Chemists' Society, 94:435-447.

Condelli, N., Caruso, M. C., Galgano, F., Russo, D., Milella, L., \& Favati, F. (2015) Prediction of the antioxidant activity of extra virgin olive oils produced in the Mediterranean area. Food Chemistry, 177:233-239.

Cook-Mills, J. M., \& Mc Cary, C. A. (2010) Isoforms of vitamin E differentially regulate inflammation. Endocrine, Metabolic \& Immune Disorders-Drug Targets, 10:348-366.

Cruz, R., \& Casal, S. (2013) Validation of a fast and accurate chromatographic method for detailed quantification of vitamin $\mathrm{E}$ in green leafy vegetables. Food Chemistry, 141:1175-1180.
Esterbauer, H., Dieber-Rotheneder, M., Striegl, G., \& Waeg, G. (1991) Role of vitamin $E$ in preventing the oxidation of low-density lipoprotein. American Journal of Clinical Nutrition, 53: 314S-321S.

European Regulation EU 1169/2011. "on the provision of food information to consumers, amending Regulations (EC) No 1924/2006 and (EC) No 1925/2006 of the European Parliament and of the Council, and repealing Commission Directive 87/250/EEC, Council Directive 90/496/EEC, Commission Directive 1999/10/EC, Directive 2000/13/EC of the European Parliament and of the Council, Commission Directives 2002/67/EC and 2008/5/EC and Commission Regulation (EC) No 608/2004." Official Journal of the European Union, L 304, 2011, pp. 18-63.

Franco, M. N., Galeano-Díaz, T., Sánchez, J., De Miguel, C., \& Martín-Vertedor, D. (2014) Total phenolic compounds and tocopherols profiles of seven olive oil varieties grown in the south-west of Spain. Journal of Oleo Science, 63:115-125.

Garcia, B., Magalhaes, J., Fregapane, G., Salvador, D. S., \& PaivaMartins, F. (2012) Potential of selected Portuguese cultivars for the production of high quality monovarietal virgin olive oil. European Journal of Lipid Science and Technology, 114:1070-1082.

García-González, D. L., \& Aparicio, R. (2010) Research in olive oil: Challenges for the near future. Journal of Agricultural and Food Chemistry, 58:12569-12577.

Huang, C. L., \& Sumpio, B. E. (2008) Olive oil, the mediterranean diet, and cardiovascular health. Journal of the American College of Surgeons, 207:407-416.

ISO 9936. (2006). Animal and vegetable fats and oils-determination of tocopherol and tocotrienol contents by high-performance liquid chromatography.

Kalogeropoulos, N., \& Tsimidou, M. Z. (2017) Antioxidants in Greek virgin olive oils. Antioxidants, 3:387-413.

Kuhn, M., \& Johnson, K. (2013) Applied predictive modeling. New York, NY: Springer Science Business Media.

Laroussi-Mezghani, S., Le Dréau, Y., Molinet, J., Hammami, M., Grati-Kamoun, N., \& Artaud, J. (2016) Biodiversity of Tunisian virgin olive oils: Varietal origin classification according to their minor compounds. European Food Research and Technology, 242: 1087-1099.

López-Miranda, J., Pérez-Jiménez, F., Ros, E., De Caterina, R., Badimón, L., Covas, M. I., ... Yiannakouris, N. (2010) Olive oil and health: Summary of the II international conference on olive oil and health consensus report, Jaén and Córdoba (Spain) 2008. Nutrition, Metabolism and Cardiovascular Diseases, 20:284-294.

Malheiro, R., Casal, S., Teixeira, H., Bento, A., \& Pereira, J. A. (2013) Effect of olive leaves addition during the extraction process of Overmature fruits on olive oil quality. Food and Bioprocess Technology, 6:509-521.

Matos, L. C., Cunha, S. C., Amaral, J. S., Pereira, J. A., Andrade, P. B., Seabra, R. M., \& Oliveira, B. P. P. (2007) Chemometric characterization of three varietal olive oils (Cvs. Cobrançosa, Madural and Verdeal Transmontana) extracted from olives with different maturation indices. Food Chemistry, 102: 406-414.

Nocella, C., Cammisotto, V., Fianchini, L., D’Amico, A., Novo, M., Castellani, V., ... Carnevale, R. (2018) Extra virgin olive oil and cardiovascular diseases: Benefits for human health. Endocrine, Metabolic \& Immune Disorders-Drug Targets, 18:4-13. https:// doi.org/10.2174/1871530317666171114121533

Noorali, M., Barzegar, M., \& Sahar, M. A. (2017) Antioxidant compounds of Iranian olive oils influenced by growing area, ripening stage, and cultivar. European Journal of Lipid Science and Technology, 119:1600029.

Pereira, J. A., Alves, M. R., Casal, S., \& Oliveira, M. B. P. P. (2004) Effect of olive fruit fly infestation on the quality of olive oil from 
cultivars Cobrançosa Madural and Verdeal Transmontana. Italian Journal of Food Science, 16:355-365.

Pereira, J. A., Casal, S., Bento, A., \& Oliveira, M. B. P. P. (2002) Influence of olive storage period on oil quality of three Portuguese cultivars of Olea europea, Cobrançosa, Madural, and Verdeal Transmontana. Journal of Agricultural and Food Chemistry, 50: 6335-6340.

Peres, F., Martins, L. L., Mourato, M., Vitorino, C., Antunes, P., \& Ferreira-Dias, S. (2016) Phenolic compounds of "Galega vulgar" and "Cobrançosa" olive oils along early ripening stages. Food Chemistry, 21:51-58.

Pryor, W. A. (2000) Vitamin E and heart disease: Basic science to clinical intervention trials. Free Radical Biology \& Medicine, 28: 141-164.

Psaltopoulou, T., Kosti, R. I., Haidopoulos, D., Dimopoulos, M., \& Panagiotakos, D. B. (2011) Olive oil intake is inversely related to cancer prevalence: A systematic review and a meta-analysis of 13800 patients and 23340 controls in 19 observational studies. Lipids in Health and Disease, 10:127.

Špika M. J., Kraljić K., \& Škevin D. (2016) Tocopherols: Chemical structure, bioactivity, and variability in Croatian virgin olive oils, products from olive tree. In Boskou, D., \& Clodoveo, M. L. (Eds.), Products from Olive Tree (pp. 317-329). Rijeka, Croatia: InTechOpen. https://doi.or/105772/64658

Tura, D., Gigliotti, C., Pedò, S., Failla, O., Bassi, D., \& Serraiocco, A. (2007) Influence of cultivar and site of cultivation on levels of lipophilic and hydrophilic antioxidants in virgin olive oils (Olea europea L.) and correlations with oxidative stability. Scientia Horticulturae, 112:108-119.

Uluata, S., Altuntas, Ü., \& Özçelik, B. (2016) Biochemical characterization of Arbequina extra virgin olive oil produced in Turkey. Journal of the American Oil Chemists' Society, 93:617-626.

Venables, W. N., \& Ripley, B. D. (2002) Modern Applied Statistics with $S$ (Statistics and Computing) (4th ed.). New York, NY: Springer.

Visioli, F., \& Bernardini, E. (2011) Extra virgin olive oil's polyphenols: Biological activities. Current Pharmaceutical Design, 17: 786-804.

Xiang, C., Xu, Z., Liu, J., Li, T., Yang, Z., \& Ding, C. (2017) Quality, composition, and antioxidant activity of virgin olive oil from introduced varieties at Liangshan. LWT-Food Science and Technology, 78:226-234. 